\title{
Ketotic Hypoglycemia in Children: A Review
}

\author{
MD. AL-AMIN MRIDHA ${ }^{1}$, ABDUL MATIN² $^{2}$
}

\begin{abstract}
Ketotic hypoglycemia is the most common form of childhood hypoglycemia. Hypoglycemic episodes typically occur during periods of intercurrent illness when food intake is limited. The term ketosis should not be confused with Ketoacidosis. Children with ketotic hypoglycemia have plasma alanine concentrations that are markedly reduced in the basal state after an overnight fast and decline even further with prolonged fasting. The classic history is of a child who has eaten poorly or misses an evening meal, is difficult to rouse from sleep the next morning, and displays neuroglycopenic symptoms that may range from lethargy to seizure. Hypoglycemic episodes are especially likely to occur during an illness, when food intake is limited. Clinical diagnosis is made by identification of ketones in plasma and urine, Whipple's triad hypoglycemia, and exclusion of endocrine/metabolic disease. It is therefore essential that appropriate investigation is performed at the time of hypoglycemia to exclude other causes This condition usually presents between the ages of 18 months to 5 years and it commonly remits spontaneously by the age of 8 to 9 years.
\end{abstract}

Keywords: Children, Ketotic hypoglycemia, alanine, gluconeogenesis.

\section{Introduction}

Ketotic hypoglycemia $(\mathrm{KH})$ is the most common phenomenon characterized by reduced fasting tolerance in children who are otherwise healthy. This condition is typically diagnosed in early childhood. Idiopathic ketotic hypoglycemia $(\mathrm{IKH})$ is similarly described as ketotic hypoglycemia and the term ketosis should not be confused with Ketoacidosis. Hypoglycemic episodes typically occur during periods of intercurrent illness when food intake is limited ${ }^{1}$. Hypoglycemia with ketosis is a diagnostic category and ketotic hypoglycemia a specific diagnosis. Ketosis accompanying new hypoglycemia suggests it has not been caused by any of the many varieties of hyperinsulinemic and reactive hypoglycemia ${ }^{2}$. A single episode of hypoglycemia and ketosis can occur during the stress and anorexia of an illness like gastroenteritis. Hypoglycemia with ketosis can also occur, sometimes repeatedly until diagnosed, from a variety of inborn errors of carbohydrate metabolism, hormone deficiencies, ingestions, and prolonged

1. Professor of Paediatrics, Department of Paediatrics, Shaheed Suhrawardy Medical College, Dhaka

2. Professor and Head, Department of Paediatrics, Shaheed Suhrawardy Medical College, Dhaka

Correspondence: Dr. Md. Al-Amin Mridha, Professor of Paediatrics, Department of Paediatrics, Shaheed Suhrawardy Medical College, Dhaka-1207. Mobile: +8801711178126, Email: drmdalamin@yahoo.com

Received: 06-04-2019

Accepted: 24-06 -2019 starvation. However, most otherwise healthy young children who suffer repeated episodes of morning hypoglycemia and ketosis will be diagnosed with ketotic hypoglycemia with no other underlying disease ${ }^{3,4}$.

\section{Pathophysiology}

Ketotic hypoglycemia has been recognized for nearly a century as a common type of childhood hypoglycemia, with a well-characterized presentation and course but an incompletely understood pathophysiology ${ }^{1,2}$. It has been hypothesized that this disorder may occur due to the failure to maintain hepatic glucose production and not increased glucose oxidation. IKH appears more pronounced in younger infants due to their limited stores and increased glucose utilization. Under fasting, younger infants have a more pronounced increase in ketone bodies and decrease in glucose versus older children. As expected, fasting tolerance improved with age thus supporting the premise that this disorder is physiologic and not pathologic.

An alternative explanation for IKH is that these patients may be exhibiting the cumulative effect of unidentified partial deficiencies of enzymes involved in glucose homeostasis. It was hypothesized that another phenotype-modifying mutation may play a role in mitochondrial fatty acid â-oxidation, that is, 
synergic heterozygosity, thus providing an alternative explanation for $I \mathrm{KH}^{1,5}$.

Children with ketotic hypoglycemia have plasma alanine concentrations that are markedly reduced in the basal state after an overnight fast and decline even further with prolonged fasting. Alanine, produced in muscle, is a major gluconeogenic precursor. Alanine is the only amino acid that is significantly lower in these children, and infusions of alanine $(250 \mathrm{mg} / \mathrm{kg})$ produce a rapid rise in plasma glucose without causing significant changes in blood lactate or pyruvate levels, indicating that the entire gluconeogenic pathway from the level of pyruvate is intact, but that there is a deficiency of substrate. Glycogenolytic pathways are also intact because glucagon induces a normal glycemic response in affected children in the fed state. The levels of hormones that counter hypoglycemia is appropriately elevated, and insulin is appropriately low.

The etiology of ketotic hypoglycemia may be a defect in any of the complex steps involved in protein catabolism, oxidative deamination of amino acids, transamination, alanine synthesis, or alanine efflux from muscle.

Ketosis represents the attempt at switching to an alternate fuel supply. Those with ketotic hypoglycemia may represent the low end of the spectrum of capacity to tolerate fasting. ${ }^{2,6}$. There are two enzymes involved in ketolysis: acetoacetate succinyl-CoA transferase which catalyzes the formation of acetoacetyl-CoA from acetoacetate, and âketothiolase which catalyzes the cleavage of acetoacetyl-CoA into two acetyl-CoA molecules. Deficiencies of either enzyme result in a defect of ketone utilization.

Children with ketotic hypoglycemia are frequently smaller than age-matched controls and often have a history of transient neonatal hypoglycemia. Any decrease in muscle mass may compromise the supply of gluconeogenic substrate at a time when glucose demands per unit of body weight are already relatively high, thus predisposing the patient to the rapid development of hypoglycemia, with ketosis representing the attempt to switch to an alternative fuel supply.

Children with ketotic hypoglycemia may represent the low end of the spectrum of children's capacity to tolerate fasting. Similar relative intolerance to fasting is present in normal children, who cannot maintain blood glucose after 30-36 hour of fasting, compared with the adult's capacity for prolonged fasting. Although the defect may be present at birth, it may not be evident until the child is stressed by more prolonged periods of calorie restriction ${ }^{1,7}$.

Moreover, the spontaneous remission observed in children at age 8-9 years might be explained by the increase in muscle bulk with its resultant increase in supply of endogenous substrate and the relative decrease in glucose requirement per unit of body mass with increasing age.

\section{Clinical Features}

The classic history is of a child who eats poorly or completely avoids the evening meal, difficult to arouse from sleep the following morning and hence eats poorly again, and may have neuroglycopenic symptoms that range from lethargy to seizure or be comatose by mid-morning. Another common presentation occurs when parents sleep late and the affected child is unable to eat breakfast, thus prolonging the overnight fast ${ }^{1}$.. Hypoglycemic episodes are especially likely to occur during an illness, when food intake is limited.

\section{Symptoms of Hypoglycemia ${ }^{3}$}

- Shakiness and dizziness.

- Sweating.

- Hunger.

- Headache.

- Irritability.

- Sudden behavioral changes, such as crying for no apparent reason.

- Clumsy or jerky movements.

- Difficulty paying attention, or confusion.

- Tingling sensations around the mouth.

Signs of Hypoglycemia ${ }^{1,8}$

- Perspiration

- Palpitation (tachycardia)

- Pallor

- Visual disturbances ("! acuity, diplopia)

- Inability to concentrate

- Paresthesia

- Somnolence, lethargy

- Seizures, Coma

- Stroke, hemiplegia, aphasia 
This condition usually presents between the ages of 18 months to 5 years and commonly remits spontaneously by the age of 8-9 years.

\section{Diagnosis and Differential Diagnosis}

Ketotic hypoglycemia should be considered only a diagnosis of exclusion, as episodic hypoglycemia with ketosis can occur with deficiencies of several hormones or a variety of defects of gluconeogenesis or glycogen metabolism, especially hypopituitarism and glycogen storage diseases ${ }^{5}$.

Clinical diagnosis is made by identification of ketones in plasma and urine, Whipple's triad hypoglycemia (Symptoms of hypoglycemia especially after fasting or heavy exercise, A low plasma glucose measured at the time of the symptoms and Relief of symptoms when the glucose is raised to normal) and exclusion of endocrine/metabolic diseases. It is therefore essential that appropriate investigation is performed at the time of hypoglycemia to exclude other causes. At the time of documented hypoglycemia, a serum glucose level less than $50 \mathrm{mg} / \mathrm{dl}$ in children ${ }^{6}$, there is associated ketonuria and ketonemia; plasma insulin concentrations are appropriately low, d"5-10 iU/mL, thus excluding hyperinsulinemia.

Blood and urine samples on arrival are important for exclusion of differential diagnosis.

Blood should be sent for measurement of the following:

- Markers of principal regulatory hormones: Insulin, ACTH, growth hormone and cortisol

- Markers of fatty acid metabolism: Ketones (betahydroxybutyrate and acetoacetate) and free carnitine

- Markers of gluconeogenic pathways: lactate, pyruvate and alanine

Urine should be sent for measurement of the following:

- Ketones

- Amino acids

- Toxicological screening- salicylates

The diagnosis of ketotic hypoglycemia is confirmed by a supervised fast. Hypoglycemia with elevated plasma free fatty acids, â-hydroxybutyrate, and acetoacetate develops within 14 to 24 hours in most of these children, whereas normal children of similar age can acquire features of hypoglycemia by 36 hours ${ }^{1,7}$.
Although older infants and children with pituitary deficiency present with ketotic hypoglycemia. However, their hypoglycemia is not responsive to diazoxide and only remits with replacement of deficient hormones (including thyroxine, as well as growth hormone and cortisol).

Hypoglycemia in growth hormone deficiency may be the result of decreased lipolysis. Hypoglycemia with cortisol deficiency may be reduced liver glycogen reserves plus diminished gluconeogenesis, owing to a failure to supply endogenous gluconeogenic substrate in the form of amino acids from muscle proteolysis. Investigation of a child with hypoglycemia therefore requires exclusion of $\mathrm{ACTH}$, cortisol, or growth hormone deficiency ${ }^{8}$.

\section{Treatment and outcome}

In anticipation of spontaneous resolution of this syndrome, treatment of ketotic hypoglycemia consists of frequent feedings of a high protein, highcarbohydrate diet. During intercurrent illnesses, parents should be taught to test the child's urine for the presence of ketones, the appearance of which precedes hypoglycemia by several hours. In the presence of ketonuria, liquids of high carbohydrate content should be offered to the child. If these cannot be tolerated, the child should be treated with intravenous glucose administration in a hospital.

Spontaneous remission by age 8 to 9 years might be explained by the increase in muscle bulk relative to brain size, with a resultant increase in the supply of endogenous substrate and the relative decrease in glucose requirement per unit of body mass with increasing age.

Episodes of ketotic hypoglycemia can be prevented or minimized by avoidance of prolonged fasting. The overnight fast should be shortened to less than 10 to 12 hours with a carbohydrate bedtime snack and prompt breakfast. When episodes have been triggered by illness, parents may test the child's urine for ketones-the appearance of which precedes the hypoglycemia by several hours and indicates a need for high-carbohydrate liquids. If these cannot be tolerated, the child should be taken to the emergency department for intravenous glucose $e^{8,9}$.

\section{Conclusion}

Ketotic hypoglycemia has been recognized for nearly a century as a common type of childhood hypoglycemia that should not be confused with 
ketoacidosis. Episodes of ketotic hypoglycemia can be prevented or minimized by avoidance of prolonged fasting. This condition remits spontaneously by the age of 8-9 yr.

\section{References:}

1. Mark A. Sperling. Hypoglycemia. In: Robert M. Kliegman, Bonita F. Stanton, Joseph W. St Geme III, Nina F. Schor, Richard E. Behrman, Editors: Nelson textbook of Pediatrics. $20^{\text {th }} \mathrm{Ed}$. Philadelphia: Elsevier; 2016. P. 773-788

2. Matthieu $\mathrm{JM}^{1}$, Boulat O. Ketotic hypoglycemia in children. Rev Med Suisse Romande. 2002 Dec;122(12):640-44.

3. Royal Berkeshire, NHS Foundation trust. Hypoglycemia and Ketototic hypoglycemia, February 2016. Available from: http:// www.royalberkshire.nhs.uk/patient-informationleaflets/Paediatrics\%20hypoglycaemia.

4. Ketotic Hypoglycemia in Wikipedia; a free encyclopedia. Last edited on 23 August 2017. Available from: https://en.wikipedia.org/wiki/ Ketotic_hypoglycemia
5. Claude Marcus, Jenny Alkeìn, Jens Eriksson, Leif Blom, Jan Gustafsson. Insufficient Ketone Body Use Is the Cause of Ketotic Hypoglycemia in One of a Pair of Homozygotic Twins. The Journal of Clinical Endocrinology \& Metabolism 2007;92(11):4080-4, https://doi.org/10.1210/ jc. 2007-0661

6. Sondheimer N. Ketotic hypoglycemia. In: Lisa $B$. Zaoutis, and Vincent W. Chiang editors. Comprehensive Pediatric Hospital Medicine. Philadelphia: Mosby Elsevier; 2007. Available from: https://www.sciencedirect.com/topics/ medicine-and-dentistry/ketotic-hypoglycemia

7. Lang TF, Hussain K. Metabolism. In: Advances in Clinical Chemistry, 2014. Available from https:/ /www.sciencedirect.com/bookseries/advancesin-clinical-chemistry

8. Langdon DR, Stanley CA,Sperling MA. Hypoglycemia in the toddler and child In: Mark A. Sperling Editor, Pediatric Endocrinology, $4^{\text {th }}$ Edition Philadelphia: Elsevier;2014. p. 920-955

9. Gallagher MP, Censani M, Oberfield SE. Hypoglycemia. In: Polin RA, Ditmar MF editors. Pediatric Secrets. $6^{\text {th }}$ ed. RELX India Pvt Ltd: Elsevier; 2016.p.208-9 